PHYSICAL REVIEW E 72, 011502 (2005)

\title{
Kinetics and mechanism of the interconversion of inverse bicontinuous cubic mesophases
}

\author{
Adam M. Squires \\ Cavendish Laboratory, Cambridge University, Cambridge CB3 OHE, United Kingdom \\ R. H. Templer and J. M. Seddon \\ Imperial College of Science, Technology and Medicine, London SW7 2AY, United Kingdom \\ J. Woenkhaus and R. Winter \\ University of Dortmund, D-44227 Dortmund, Germany \\ T. Narayanan and S. Finet \\ ESRF, F-38043 Grenoble, France
}

(Received 2 March 2005; published 11 July 2005)

\begin{abstract}
This paper describes time-resolved x-ray diffraction data monitoring the transformation of one inverse bicontinuous cubic mesophase into another, in a hydrated lipid system. The first section of the paper describes a mechanism for the transformation that conserves the topology of the bilayer, based on the work of Charvolin and Sadoc, Fogden and Hyde, and Benedicto and O'Brien in this area. We show a pictorial representation of this mechanism, in terms of both the water channels and the lipid bilayer. The second section describes the experimental results obtained. The system under investigation was 2:1 lauric acid: dilauroylphosphatidylcholine at a hydration of $50 \%$ water by weight. A pressure-jump was used to induce a phase transition from the gyroid $\left(Q_{I I}^{G}\right)$ to the diamond $\left(Q_{I I}^{D}\right)$ bicontinuous cubic mesophase, which was monitored by time-resolved x-ray diffraction. The lattice parameter of both mesophases was found to decrease slightly throughout the transformation, but at the stage where the $Q_{I I}^{D}$ phase first appeared, the ratio of lattice parameters of the two phases was found to be approximately constant for all pressure-jump experiments. The value is consistent with a topologypreserving mechanism. However, the polydomain nature of our sample prevents us from confirming that the specific pathway is that described in the first section of the paper. Our data also reveal signals from two different intermediate structures, one of which we have identified as the inverse hexagonal $\left(H_{I I}\right)$ mesophase. We suggest that it plays a role in the transfer of water during the transformation. The rate of the phase transition was found to increase with both temperature and pressure-jump amplitude, and its time scale varied from the order of seconds to minutes, depending on the conditions employed.
\end{abstract}

DOI: 10.1103/PhysRevE.72.011502

PACS number(s): 61.10.-i, 61.30.St, 82.70.Kj, 64.70.Nd

\section{INTRODUCTION}

\section{A. Inverse bicontinuous cubic phases}

Aqueous dispersions of lipids form mesophase structures with a wide range of different geometries, many of them based on lipid bilayers, the basic structural element of biological membranes. While the bilayer often adopts a relatively simple geometry, as in unilamellar or multilamellar vesicles, some systems also form the more complex inverse bicontinuous cubic phases $\left(Q_{I I}\right)$. Here, a single continuous bilayer separates two continuous networks of water channels. The bilayer midplane lies on a mathematical surface known as a triply periodic minimal surface (TPMS) [1-3]. Three $Q_{I I}$ phases are known in lipid systems, based on the Schwarz primitive (P) and diamond (D) and the Schoen gyroid (G) TPMSs. They are therefore known as the $Q_{I I}^{P}, Q_{I I}^{D}$, and $Q_{I I}^{G}$ phases, and they have crystallographic space groups $\operatorname{Im} 3 m, P n 3 m$, and $I a 3 d$, respectively.

The TPMS for each $Q_{I I}$ phase is shown in Fig. 1. In order to aid visualization, the phases are often represented as "skeletal graphs"-networks of rods which simply represent the centers of the water channels. These are also shown in Fig. 1. The $Q_{I I}^{P}$ phase has two identical networks of water channels.
The water channels in each network meet at $90^{\circ}$ in six-way junctions. In the $Q_{I I}^{D}$ phase, the water channels meet in fourway junctions at the tetrahedral angle of $109^{\circ}$. Again, the two water regions are identical. In the $Q_{I I}^{G}$ the water channels meet in three-way junctions at $120^{\circ}$. Unlike in the other two $Q_{I I}$ mesophases, the two water regions in the $Q_{I I}^{G}$ phase are no longer identical. Each has a chirality associated with it, and the two regions are enantiomeric.

Such structures have very specific and controllable water channel sizes and a large membrane area per unit volume. These properties give rise to the use of $Q_{I I}$ phases in a number of recent applications, including protein crystallization [4] and drug delivery [5], and offer potential roles in ultrafiltration and biosensors [6]. In addition, inverse bicontinuous cubic phases have been found to perform a number of biological roles, and it is thought that similar structures act as intermediates in the mechanism of membrane fusion and fission.

\section{B. Mechanisms for the interconversion of $Q_{I I}$ phases}

An interesting property of the three TPMSs considered here is that they are all topologically equivalent, and may be 

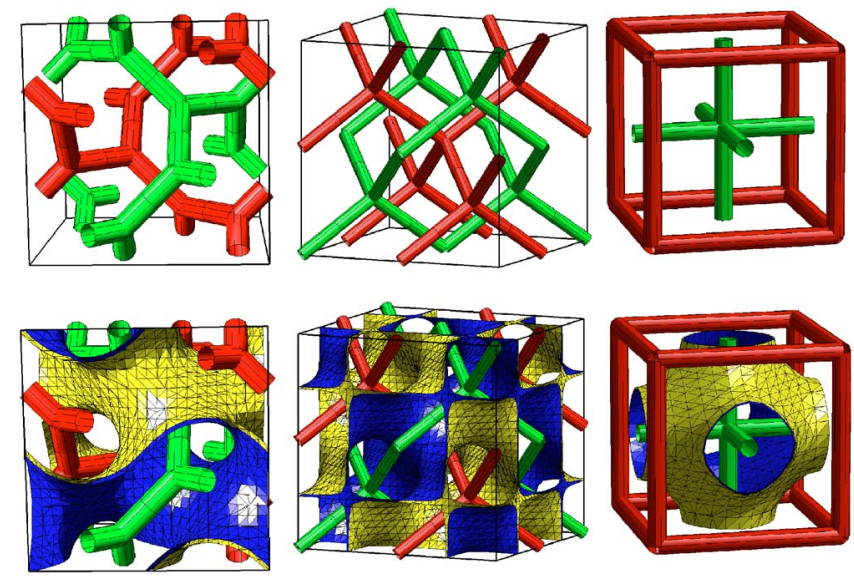

FIG. 1. (Color online) Different descriptions of the inverse bicontinuous cubic phases. Top: Skeletal graphs representing the centers of the water channels. Bottom: Skeletal graphs and surfaces that approximate the underlying TPMSs. The three inverse bicontinuous cubic phases shown are (from left) $Q_{I I}^{G}, Q_{I I}^{D}$, and $Q_{I I}^{P}$. One unit cell of each structure is shown for the $Q_{I I}^{G}$ and $Q_{I I}^{P}$ phases, and eight of the crystallographic unit cells of the $Q_{I I}^{D}$ phase. The two networks of rods and the two sides of the surface have been shaded differently for clarity - the shading is not intended to represent any physical difference.

interconverted by a process known as the Bonnet transformation. This transformation leaves the Gaussian curvature $K$ at any point on the TPMS unchanged. It also preserves all angles, distances, and areas on the surface [7]. Although this mechanism may in theory interconvert the underlying TPMSs, in reality it is unlikely that transitions between actual $Q_{I I}$ phases occur via such a mechanism. This is because the Bonnet transformation requires that parts of the minimal surface pass through one another; in a $Q_{I I}$ phase this would require regions of the lipid bilayer to be torn apart and then joined back together again [8], which is probably physically unrealistic.

However, the fact that the underlying TPMSs of any two $Q_{I I}$ phases may be related by the Bonnet transformation is nonetheless significant, even if this is not the actual mechanism of their interconversion. It is still the case that the two $Q_{I I}$ phases are topologically equivalent, and therefore that one phase could in principle be transformed into the other without tearing the lipid bilayer. It is also true in such a case that the Gaussian curvatures and areas of equivalent parts of the TPMS of each $Q_{I I}$ phase are equal, and so the two $Q_{I I}$ phases will have the same monolayer bending energy, so long as the bilayer is thin with respect to the radii of curvature of the TPMS $[9,10]$. For two TPMSs to be related by the Bonnet transformation, they will have a certain ratio of lattice parameters. This ratio takes the value of $a(G) / a(D)$ $=1.576$ or $a(P) / a(D)=1.279[11]$.

Descriptions of mechanisms which can interconvert $Q_{I I}$ phases without tearing the lipid bilayer have been developed both in terms of the "skeletal graphs" which lie at the centers of the water channels, and in terms of the TPMSs which lie at the bilayer midplane (Fig. 1). Sadoc and Charvolin [12] have considered the transformations in terms of "merging" junctions on the skeletal graphs, where the water channels meet. Thus for example each four-way junction in the $Q_{I I}^{D}$ phase may be formed by merging two adjacent three-way junctions from the $Q_{I I}^{G}$ phase. Similarly, each six-way junction from the $Q_{I I}^{P}$ phase may be formed by merging two adjacent four-way junctions in the $Q_{I I}^{D}$ phase. (The reverse transformations may be brought about by "pulling apart" one junction into two, with the formation of a new connecting water channel.) Benedicto and O'Brien [13] have suggested a more global description in terms of the overall "labyrinthine nets" representing the skeletal graphs. Fogden and Hyde [14] have instead considered continuous distortions of the TPMS lying at the middle of the lipid bilayer. They suggest a number of mechanisms for the interconversion of $Q_{I I}$ phases which not only conserve topology, but convert one TPMS to another through intermediate surfaces which are themselves minimal surfaces. In other words, through all intermediate structures, the middle of the bilayer itself has a mean curvature of zero throughout. The work has been extended [15] to include a description of the $Q_{I I}^{P}-Q_{I I}^{D}$ transformation in terms of a "medial graph" obtained from the medial surface-a more rigorously defined geometric construct equivalent to the skeletal graphs.

\section{Previous experimental work}

Although a considerable amount of work has been done on the thermodynamic properties of the $Q_{I I}$ phases $[9,16,17]$, little experimental evidence is available on the actual kinetics or mechanism of their intertransformation. The transformation appears to proceed with no measurable enthalpy change, which has been cited as evidence that no disruption of bilayer integrity occurs [18]. It has also been noted for many systems $[9,18]$ that the ratio of lattice parameters of $Q_{I I}$ phases at the phase boundary is close to that predicted by the Bonnet relationship [10]. We have obtained time-resolved data on the transition from $Q_{I I}^{G}$ to $Q_{I I}^{D}$ for the 2:1 lauric acid:dilauroylphosphatidylcholine (2LA-DLPC) system and found the kinetics to be fast $(<0.5 \mathrm{~s})$ [19].

\section{MODELING THE INTERTRANSFORMATION OF $Q_{I I}$ PHASES}

In this section we show a pictorial representation of a complete mechanism for both the $Q_{I I}^{P} Q_{I I}^{D}$ and $Q_{I I}^{D} Q_{I I}^{G}$ interconversions, based on the earlier work described in the previous section. We describe the transformation in the $\mathrm{P} \rightarrow \mathrm{D}$ and $\mathrm{D} \rightarrow \mathrm{G}$ directions, so we will talk of pulling apart junctions in the skeletal graphs representing the water channels. From this point of view, we therefore suggest that the $Q_{I I}^{P}$ - $Q_{I I}^{G}$ transformation would simply proceed via an intermediate $Q_{I I}^{D}$ phase.

We consider each transformation to involve three processes.

(1) Rearrangement within the unit cell. This involves the pulling apart or merging of junctions in the skeletal graph.

(2) Change in shape of the unit cell. This is a simple tetragonal or rhombohedral distortion of the overall structure, which changes either the ratio of the unit cell dimensions or else the angles of the unit cell. 
(3) A further rescaling, changing the overall size of the unit cell, required to take into account considerations such as the conservation of bilayer area or volume fraction. Such considerations will be discussed later.

The distinction between these three processes is not intended to suggest any sequential relationship; the three processes probably occur concomitantly throughout the transformation. The distinction is a conceptual one, made to help visualize the process.

Because neither transformation simply converts one conventional unit cell of one $Q_{I I}$ phase into one unit cell of another, we will be defining a new "repeat cell" for each transformation, common to both the initial and final structures, to describe intermediate stages in the transformation. We note that this may not necessarily correspond to the smallest possible crystallographic unit cell for the intermediate structures.

The $Q_{I I^{-}}^{D} Q_{I I}^{G}$ and $Q_{I I^{-}}^{P} Q_{I I}^{D}$ transformations will now be described in the next two sections, in light of these considerations.

\section{A. The $Q_{I I}^{D} Q_{I I}^{G}$ transformation}

The repeat cell for this transformation begins as a cube consisting of eight of the crystallographic unit cells of the $Q_{I I}^{D}$ phase. The rearrangement of water channels within this repeat cell is brought about by pulling apart each four-way junction in either the $x$ or the $y$ direction, with the creation of a new water channel each time. This is shown in the skeletal graph representation in Fig. 2. Adjacent junctions in a given water channel network are pulled apart in different directions, and the choice of which junctions are pulled in the $x$ and which in the $y$ direction imparts a chirality. This rearrangement makes two previously identical water channel networks become enantiomeric.

Simply rearranging the skeletal graphs in the repeat cell in this way produces a $Q_{I I}^{G}$ phase which has been stretched along the $z$ axis. Another process, changing the shape of the repeat cell by a tetragonal distortion by a factor of $\sqrt{2}$, produces an undistorted $Q_{I I}^{G}$ phase. This is also shown in Fig. 2. The repeat cell now contains two unit cells of the $Q_{I I}^{G}$ phase, rotated by $45^{\circ}$ about the $z$ axis.

Figure 2 also shows a portion of the surface that represents the bilayer midplane in the intermediate structures. The formulas for these are given in the Appendix. These are, in general, not minimal surfaces; in the intermediate structures between the initial and final $Q_{I I}$ structures, the middle of the bilayer may be distorted away from zero mean curvature.

As we have mentioned before, we do not intend to imply that the rearrangement and the distortion of the unit cell occur sequentially and in this order. The tetragonal distortion of the repeat cell could occur first, for example, followed by the rearrangement of the water channels within the repeat cell. It is more probable that they occur together, with a continuous combination of the two processes taking one of an infinite number of paths leading from the $Q_{I I}^{D}$ to the $Q_{I I}^{G}$ phase in Fig. 2. We suggest that one such path corresponds to the case where the bilayer midplane remains a minimal surface throughout, and therefore corresponds to the path suggested by Fogden and Hyde [14] and Schröder et al. [15]. We would expect this path to be energetically favourable because the curvature elastic energy of any transitional structures will be close to a minimum if the bilayer midplane is minimal.

From Fig. 2 we can see the position and orientation of the conventional $Q_{I I}^{G}$ unit cell relative to the repeat cell defined in our description. This would also suggest its orientation relative to the original $Q_{I I}^{D}$ phase.

\section{B. The $Q_{I I}^{P}-Q_{I I}^{D}$ transformation}

The repeat cell for this transformation begins as a cube simply consisting of one unit cell of the $Q_{I I}^{P}$ phase. The rearrangement of water channels within this repeat cell is brought about by pulling every six-way junction apart in the same direction, which in this case is along the body diagonal of the cube, that is, in the $(1,1,1)$ direction. This rearrangement produces a $Q_{I I}^{D}$ phase which has been rhombohedrally squashed in the $(1,1,1)$ direction. The cubic repeat cell now has to be stretched by a factor of 2 in this direction. This produces a rhombohedral repeat cell of the undistorted $Q_{I I}^{D}$ phase, which has been rotated by $60^{\circ}$ about the $(1,1,1)$ axis. The rhombohedral repeat cell has a quarter of the volume of the repeat cell for the $Q_{I I}^{D}$ to $Q_{I I}^{G}$ transformation described in the previous section. This is shown in Fig. 3. As before, the rearrangement of water channels within the repeat cell and the rhombohedral distortion probably do not occur as separate sequential processes. Again, Fig. 3 shows the position and orientation of a conventional unit cell of the final $Q_{I I}^{D}$, relative to the defined repeat cell for the transformation, and therefore relative to the initial $Q_{I I}^{P}$ unit cell.

\section{Surface area considerations}

In order for one such transformation to occur with conservation of topology, the total number of repeat cells must be conserved. The total amount of lipid matter in an actual lipid mesophase transformation is also conserved. This implies that the amount of lipid material in one repeat cell, which to a first approximation we consider to be proportional to the area of the TPMS, must be the same at the start and end of the transformation. This is only the case when the initial and final TPMSs are related by the Bonnet transformation, in which case they will have a certain ratio of lattice parameters. This ratio takes the value of $a(G) / a(D)=1.576$ or $a(P) / a(D)=1.279$ [11].

In the transformations as they have been described thus far, we have not imposed this constraint, and have allowed the area of the TPMS in a repeat cell to change. The initial and final TPMSs in the transformations simply as described in Secs. II A and II B have the lattice parameter ratios $a(G) / a(D)=\sqrt{2}$ and $a(P) / a(D)=1$. In order to satisfy the conservation of overall topology, we therefore introduce the third process - a further rescaling of the overall repeat cell size, which gradually occurs over the course of the transformation. This accompanies the two other processes described in the previous section (rearrangement within the cell, and change in cell shape) so as to maintain a constant TPMS area within the cell throughout. The cell is scaled up by a factor 

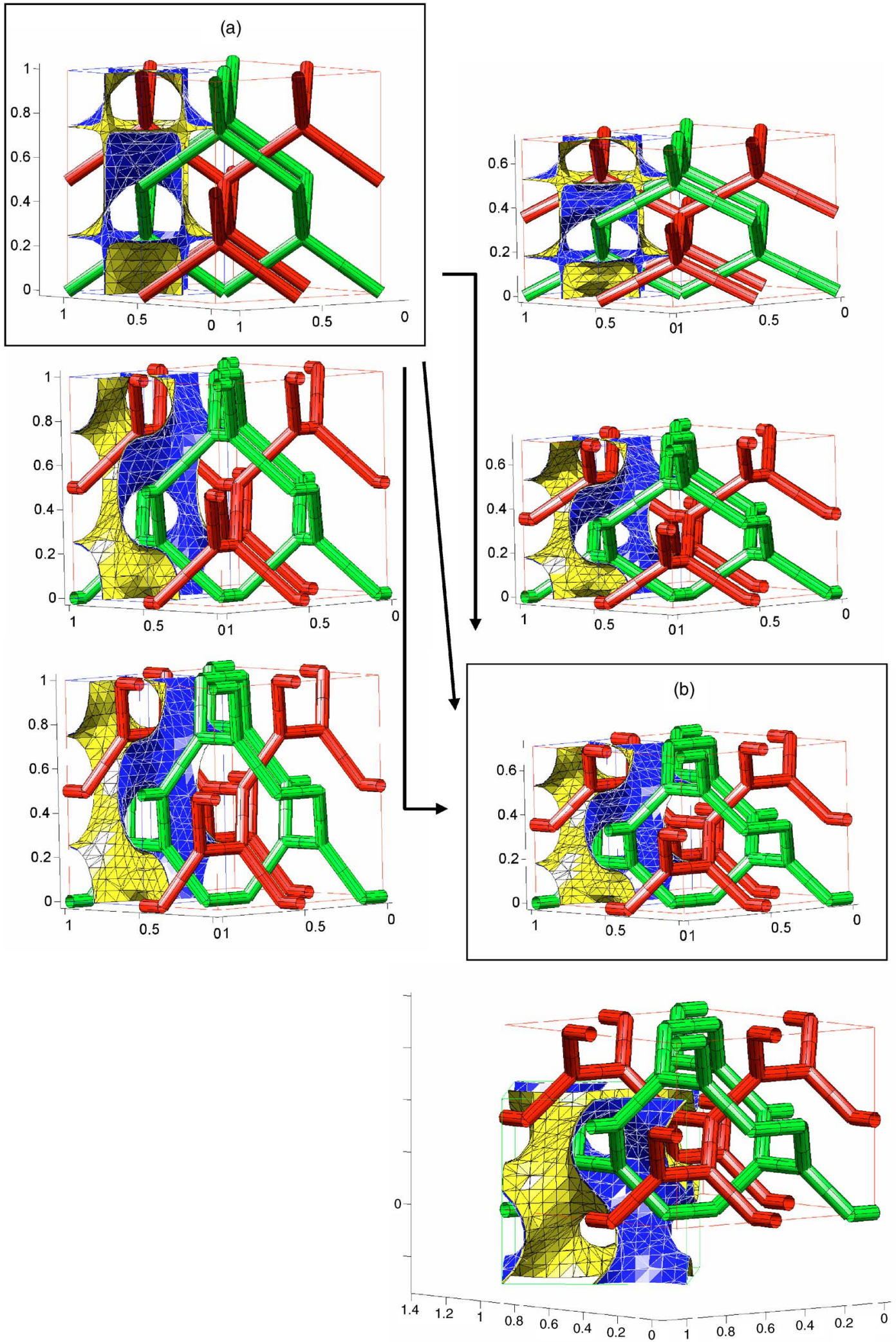

FIG. 2. (Color online) Transformation from $Q_{I I}^{D}$ (a) to $Q_{I I}^{G}$ (b). The transformation may take one of a continuum of routes, each consisting of a combination of two processes; a rearrangement within the repeat cell (top to bottom), and a tetragonal distortion of the repeat cell (left to right). Below: The final $Q_{I I}^{G}$ structure (b) showing a different section of the bilayer midplane corresponding to the conventional cubic $Q_{I I}^{G}$ unit cell. 


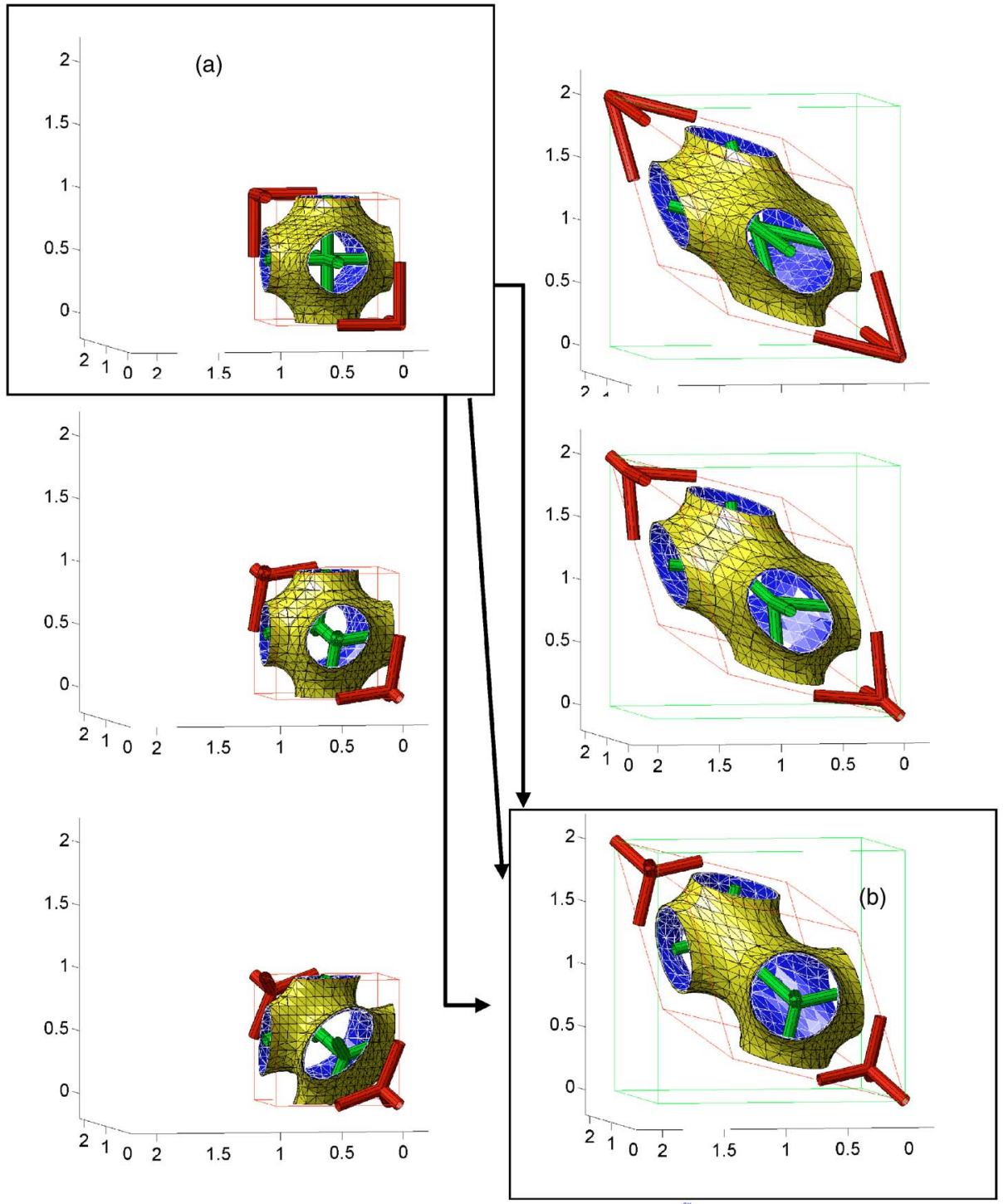

FIG. 3. (Color online) Transformation from $Q_{I I}^{P}$ (a) to $Q_{I I}^{D}(\mathrm{~b})$. The transformation consists of a combination of two processes: a rearrangement within the repeat cell (top to bottom), and a rhombohedral distortion of the repeat cell (left to right). Below: The final $Q_{I I}^{D}$ structure (b) together with a more extended section of the bilayer mid-plane, corresponding to the eight-unit-cell cube shown in Fig. 1.

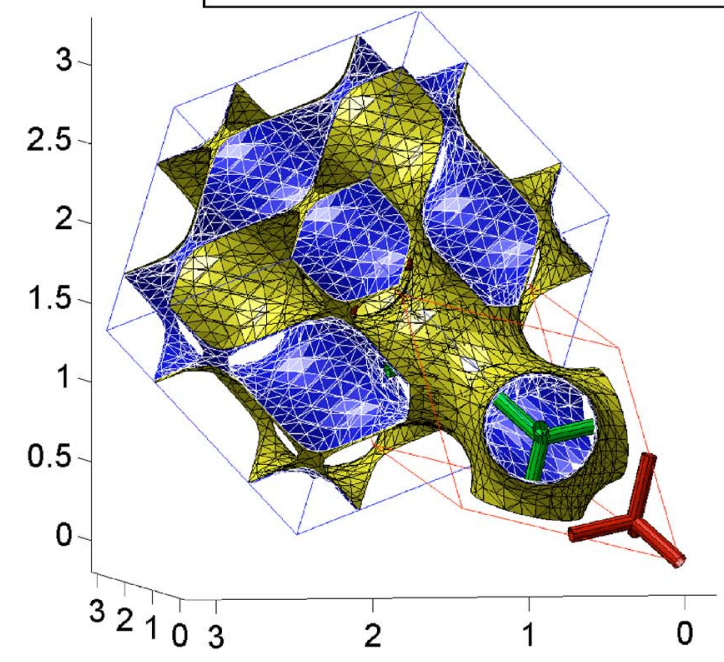

that begins with a value of 1 , and ends with a value of $\sqrt{2} / 1.576=1.1144$ for the $Q_{I I}^{G}$ to $Q_{I I}^{D}$ or $1 / 1.279=0.7819$ for the $Q_{I I}^{P}$ to $Q_{I I}^{D}$ transformation.

In summary, the suggested mechanism has four potentially measurable implications.

(1) At the first instant when a new $Q_{I I}$ phase is formed, the ratio of its lattice parameter to that of the preceding $Q_{I I}$ phase is always the same, with the values mentioned earlier, of $a(G) / a(D)=1.576$ or $a(P) / a(D)=1.279$.

(2) The different $Q_{I I}$ phases fill space differently, due to the different dimensionless areas of their underlying minimal surfaces. It is therefore not possible for a transformation be- 
tween two $Q_{I I}$ phases to conserve both topology and water content throughout. If a transformation at first proceeds through the topology-conserving mechanism described in this paper, the discrepancy in water content between the initial and final phases will result in either an excess or a deficit of water in some form or other, somewhere else in the sample. This may well produce observable effects such as new transient structures if the transformation is carried out under limiting-hydration conditions, as in the experiments described here, rather than excess water. Furthermore, under such circumstances, the transformation would eventually require a change in lattice parameter of the final phase, from the value corresponding to topological equivalence with the initial phase to a final value where its water content is the same as that of the sample as a whole. This second process will require a change in topology.

(3) The transformations described in this section imply a relationship between the orientation of a crystallite of the initial phase and that of the final phase. These are shown graphically in Figs. 2 and 3. In the $Q_{I I}^{P} Q_{I I}^{D}$ transformation, the phases are rotated relative to one another by $60^{\circ}$ about a common $(1,1,1)$ axis, while in the $Q_{I I}^{D} Q_{I I}^{G}$ transformation, the phases are rotated relative to one another by $45^{\circ}$ about a common $(0,0,1)$ axis.

(4) These descriptions also imply a change in the aspect ratio of a single crystallite. In the $Q_{I I}^{P} Q_{I I}^{D}$ transformation, the ratio of the $(1,1,1)$ dimension of the crystallite relative to a dimension in a perpendicular direction increases by a factor of 2 when going from $\mathrm{P}$ to $\mathrm{D}$. In the $Q_{I I}^{D} Q_{I I}^{G}$ transformation, the ratio of the $(0,0,1)$ dimension relative to a perpendicular dimension decreases by a factor of $\sqrt{2}$. Of course, it is possible that some subsequent rearrangement of unit cells within a crystallite occurs that changes the aspect ratio of the crystallite without a further change in topology, in which case the change might not appear to occur as predicted.

Of these four implications, the first two may be measured or observed in a polydomain system, where data are averaged over many single-domain crystallites in many different powder-averaged orientations, as is this case for the experimental data described in this paper. In Sec. IV we show results consistent with these predictions.

Experiments carried out on such systems cannot, however, determine the relative orientations of the initial and final crystals from a single domain, nor can they detect a change in its aspect ratio. Such information requires either a method of producing a completely aligned macroscopic sample consisting of one single crystal of lipid $Q_{I I}$ phase, or else an analytical technique which can probe a single microscopic crystallite. The third and fourth implications therefore remain as predictions that may be confirmed or rejected when such data become available.

\section{MATERIALS AND METHODS}

\section{A. Sample preparation}

The system under investigation was a mixture of dilauroylphosphatidylcholine (DLPC) and lauric acid (LA) in the molar ratio 2:1 LA:DLPC, hydrated to $50 \%$ water by weight. The LA and DLPC were obtained from Avanti Polar Lipids
(Alabaster, AL) and Fluka respectively, each at $>99 \%$ purity, according to the manufacturers. The purity of both lipids was confirmed by thin-layer chromatography. The lipids were left overnight in a desiccator before use to remove residual moisture. The 2LA-DLPC mixture was prepared by codissolving the appropriate ratio of LA and DLPC in cyclohexane (BDH chemical supplies, Dorset, U.K.), and removing the solvent by freezing to liquid nitrogen temperature and then freezedrying overnight. To this was added an equal mass of millipore-filtered distilled water, and this mixture was incubated overnight at $42{ }^{\circ} \mathrm{C}$, approximately $10{ }^{\circ} \mathrm{C}$ above the chain melting temperature. It was then allowed to cool, before being homogenized. Homogenization was achieved by using a centrifuge to force the sample backward and forward through a $1 \mathrm{~mm}$ diameter hole in a Teflon "hourglass." The sample was centrifuged through this device approximately ten times, until it appeared homogeneous upon visual inspection.

\section{B. Sample cell}

The experiments employed a high-pressure cell with flat diamond windows of thickness $0.7 \mathrm{~mm}$, which was designed and built in the University of Dortmund. The samples were placed in the pressure cell in Teflon rings of thickness $1 \mathrm{~mm}$, with Kapton windows held on with double-sided sticky tape. The cell is suitable for studies up to pressures of $5 \mathrm{kbar}$ and temperatures up to $140{ }^{\circ} \mathrm{C}$ with an accuracy of \pm 5 bar and $\pm 0.2^{\circ} \mathrm{C}$. The pressure jump that induced the $Q_{I I}^{G} Q_{I I}^{D}$ transition was triggered by the computer-controlled opening of an air-operated valve. This allowed a rapid jump in pressure; the cell can achieve pressure jumps of up to $1 \mathrm{kbar}$ in under 7 $\mathrm{ms}$, which is much faster than the time scale of the transformation (from seconds to minutes; see Sec. IV E). No adiabatic temperature change was detected in these experiments within the accuracy of our temperature measurements $\left( \pm 0.2^{\circ} \mathrm{C}\right)$. Further details of the apparatus are provided elsewhere [20].

\section{X-ray beamline}

Experiments at ESRF were performed at the highbrilliance beamline ID02 (experiment LS1560). Details of beam and detector specifications can be found at the ESRF website [27]. The experiments were carried out using a beam of approximate wavelength $\lambda=1.0 \AA$, with dimensions 0.46 $\times 0.15 \mathrm{~mm}^{2}$ and a typical flux of $8 \times 10^{12}$ photons per second. Two-dimensional diffraction patterns were recorded on a detector consisting of a Thomson $\mathrm{x}$-ray intensifier $\mathrm{TH}$ 49-427) lens coupled to the ESRF-developed FReLoN charge-coupled device (CCD) camera. This detector had an active area of size $230 \mathrm{~mm}$ and a frame rate of ten images $(1024 \times 1024$ pixels $)$ per second with a nominal dynamic range of 14 bits. The sample-detector distance was set to $2.000 \mathrm{~m}$. Intensity data were normalized to compensate for fluctuations in beam intensity.

\section{Time-resolved acquisition}

Time-resolved $\mathrm{x}$-ray diffraction data were obtained as a sequence of exposures, each of duration $0.1 \mathrm{~s}$, with a delay 

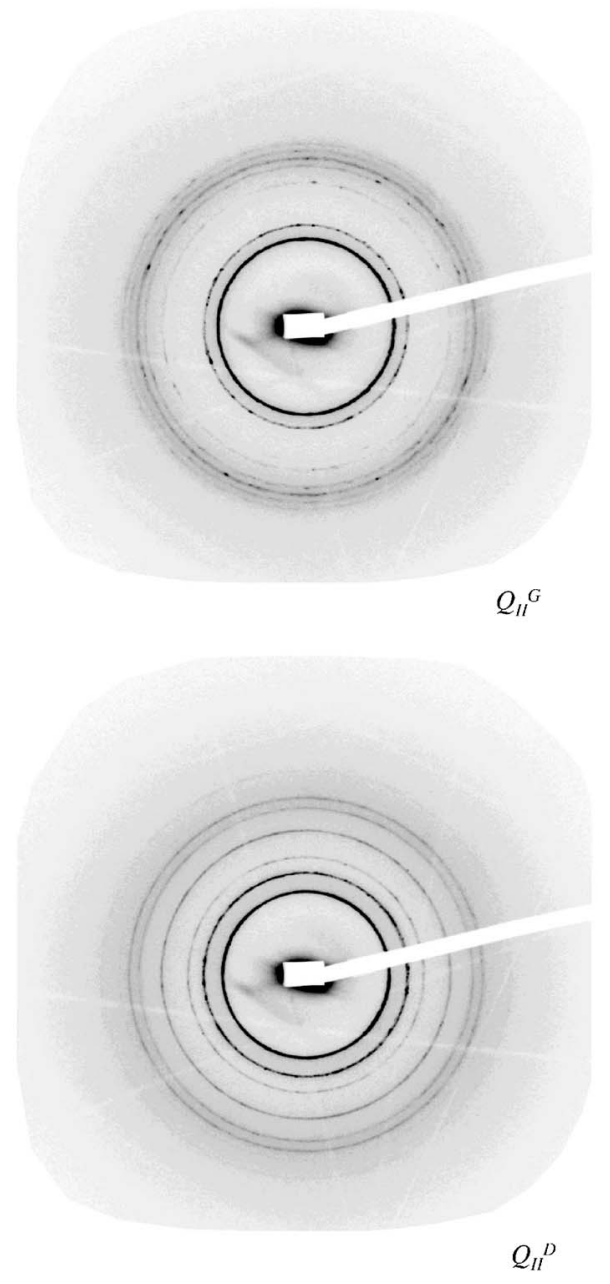

FIG. 4. Representative 2D diffraction patterns for the $Q_{I I}^{D}$ and $Q_{I I}^{G}$ phases.

between successive exposures. The delay was increased throughout the data acquisition, following a geometric progression of the form $t_{n}=t_{1} a^{n-1}$ where $t_{n}$ is the delay following exposure number $n$; our experiments used an initial delay of $t_{1}=0.1 \mathrm{~s}$ and typically a common ratio of $a=1.2$ or 1.25 .

Such a timing sequence takes exposures more rapidly earlier on in the phase transition, and progressively less frequently as time progresses. This enables us to monitor changes over a range of time scales.

\section{E. X-ray diffraction pattern analysis}

The CCD camera recorded two-dimensional (2D) diffraction patterns. As the samples were powderlike, each took the form of a series of concentric rings. Representative diffraction patterns from the $Q_{I I}^{G}$ and $Q_{I I}^{D}$ phases are shown in Fig. 4. The center of the beam was located using software developed in house. Each diffraction pattern was then integrated radially about this position to give an average pixel intensity as a function of distance from the beam center, in the form of a 1D diffraction pattern. We can convert values for the position of a reflection in pixels into a $d$ spacing, using values for sample-detector distance $l$, wavelength $\lambda$, and pixel size $p$ of

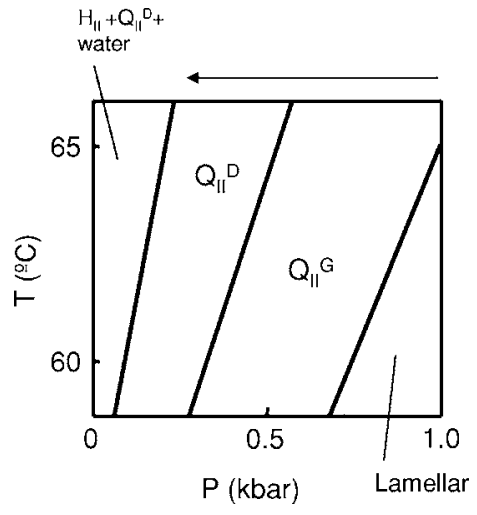

FIG. 5. $p-T$ phase diagram for 2LA-DLPC at $50 \%$ water by weight. The data were obtained at each temperature from high to low pressure, and the phase diagram is really only applicable in this direction, as there is the possibility of hysteresis if a phase boundary is approached from the low-pressure direction. Adapted from [19].

\section{$l=2000 \mathrm{~mm}, \lambda=0.9887 \AA$, and $p=0.173 \mathrm{~mm}$.}

For ease of visualization, we have taken all of the 1D diffraction patterns from a single pressure-jump experiment and displayed them displaced vertically to give a stacked plot as shown in Fig. 6 below. For greater clarity, the intensity axis is displayed on a logarithmic scale.

\section{RESULTS AND DISCUSSIONS}

\section{A. Pressure-temperature phase diagram}

The pressure-temperature phase diagram for 2LADLPC-50 wt \% water has already been obtained for pressures from 1 to 1000 bar and temperatures between 57 and $67{ }^{\circ} \mathrm{C}$ from SAXS data taken at the DESY synchrotron facility, Hamburg. The phase diagram, which we have already published in a previous article [19], is shown in Fig. 5. Before the pressure-jump experiments described here were carried out, static measurements were taken at $59.5^{\circ} \mathrm{C}$ at pres-

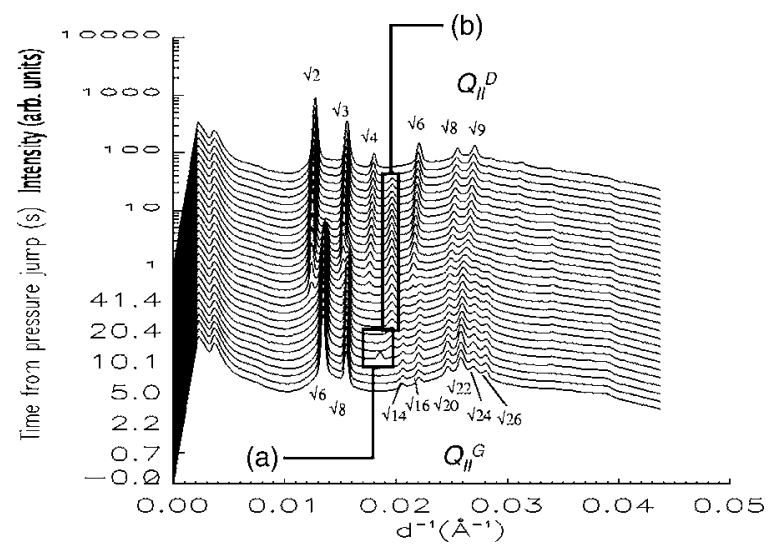

FIG. 6. Stacked plot showing a $Q_{I I}^{G}$ to $Q_{I I}^{D}$ transformation. The pressure jump was carried out at $T=59.5{ }^{\circ} \mathrm{C}$ from 600 to 240 bar. The "intensity" axis is plotted on a logarithmic scale. A short-lived (a) and a longer-lived (b) intermediate peak can clearly be seen. The viewing direction is coplanar with both the time and intensity axes, so both appear vertical in the figure. 
sures from 2000 down to 200 bar. This confirmed that the present samples showed the same phase behavior.

\section{B. Overview}

Seven pressure-jump experiments were carried out, at a variety of different temperatures and initial and final pressures. The data from all of the pressure jumps showed the same qualitative features. A typical stacked plot is shown in Fig. 6. A number of features, common to all pressure-jump data, can be seen in this figure.

(1) The system begins in the $Q_{I I}^{G}$ phase. Following the pressure jump, the set of peaks from the $Q_{I I}^{G}$ phase gradually disappears. At the same time, another set of peaks corresponding to the emergent $Q_{I I}^{D}$ phase appears.

(2) Accompanying this transformation, both sets of peaks show a very slight shift toward a higher angle, corresponding to a decrease in the lattice parameter of each phase. This is more noticeable for the $Q_{I I}^{D}$ phase.

(3) Two intermediate peaks appear and then disappear in every experiment: an earlier extremely short-lived "blip" which appears at a $d$ spacing of between 53 and $55 \AA$, and a longer-lived peak which first appears just after the disappearance of the first peak, at a $d$ spacing of between 50 and $52 \AA$. In some cases additional peaks could be seen at wider angles, accompanying these intermediate peaks. These are too small to be seen clearly in Fig. 6 . They are discussed further in the next section, and possible identities for the intermediate structures giving rise to all of these peaks are suggested.

\section{Identity of intermediates}

The longer-lived intermediate peak was accompanied by a much smaller peak at around $29 \AA$ A. In some cases, especially where the intensity was greater, it was sometimes possible to discern a third peak, at around $26 \AA$. However, this peak was very small, and generally lay on top of a position where the data showed a kink, an artefact arising from the radial integration procedure (see Fig. 7). Although the exact positions of the peaks varied between different pressure-jump experiments, in each case the longer-lived intermediate peaks always occurred with $d$ spacing ratios of exactly $1: \sqrt{3}$ to within $0.1 \AA$ (or $1: \sqrt{3}: 2$ for those cases where the position of the third peak could be determined). The ratio of $1: \sqrt{3}: 2$ is the same as that of the first three observed reflections from a phase with hexagonal symmetry; the positions of the longer-lived intermediate peaks are consistent with a hexagonal phase of lattice parameter $a=61-63 \AA$. The inverse hexagonal $H_{I I}$ phase is in fact an equilibrium phase for the 2LADLPC system at higher temperatures, lower pressures, or lower hydrations (see Fig. 5 and Refs. [19,21]). It typically appears with a slightly smaller lattice parameter of around 58 $\AA$ (this value itself becomes lower for samples with water volume fractions below about 0.3). However, these values were obtained for samples investigated at atmospheric pressure; the agreement is good enough for us to suggest that the longer-lived intermediate signals are from an $H_{I I}$ phase.

We note that it is unlikely for the transformation from the $Q_{I I}^{G}$ to the $Q_{I I}^{D}$ phase, both of which have bilayer structures, to

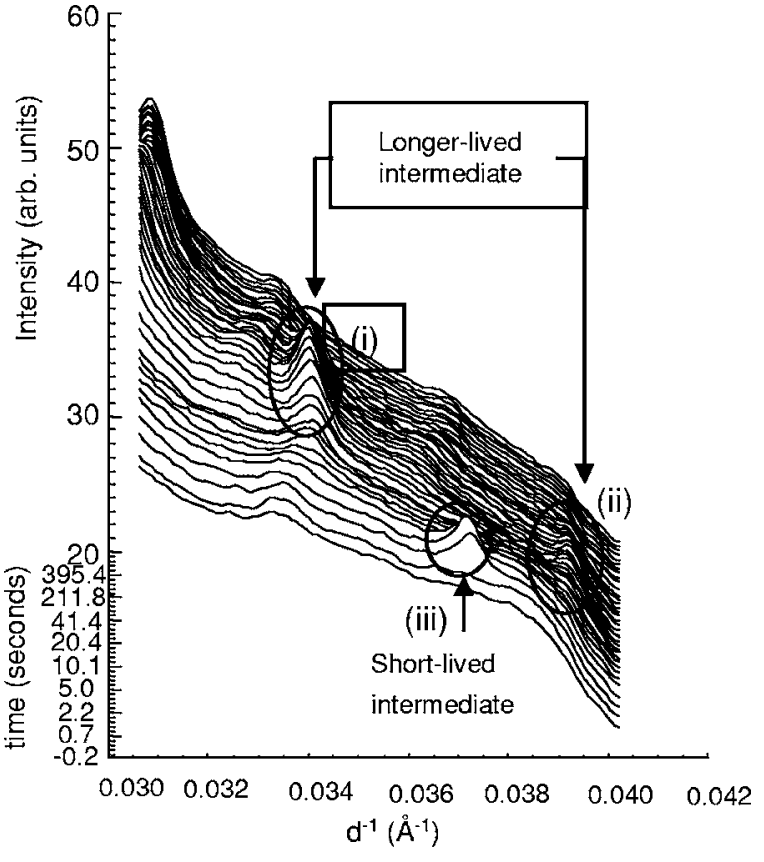

FIG. 7. Close-up of data taken over the slightly wider angle region, showing accompanying intermediate peaks. Peaks (i) and (ii) accompany the longer-lived intermediate. Peak (iii) accompanies the shorter-lived blip. The pressure jump was carried out at $T$ $=59.5{ }^{\circ} \mathrm{C}$ from 640 to 230 bar.

actually proceed via an $H_{I I}$ phase, which consists of cylindrical monolayers. Such a transformation would involve considerable energetically costly disruption of topology, as compared with the continuous transformations described in Sec. II. Instead, we suggest an alternative role for the $H_{I I}$ intermediate, relating to the water contents of the different participating phases. This is discussed further at the end of the next section.

A closer examination was made of the same wider-angle region around $d=20-30 \AA$ for diffraction patterns where the shorter-lived blip was present, to find if any similar accompanying peaks could be found at $\sqrt{3}$ and two times the $d$ spacing of this intermediate peak as well. No peak could be found at all in the $\sqrt{3}$ position. However, in some cases where the intensity of the blip was greater, a second peak could be seen at exactly twice the lattice parameter, to within $\pm 0.1 \AA$. We can therefore rule out the possibility of this corresponding to another $H_{I I}$ phase with a smaller lattice parameter; the $\sqrt{3}$ reflection in the $H_{I I}$ phase is greater in intensity than the 2 reflection for 2LA-DLPC, so it is unlikely that we should be able to observe the latter and not the former (see Fig. 7). Reflections with $d$ spacings at 54 and $27 \AA$ are instead consistent with the first two reflections from a lamellar phase of lattice parameter $a=54 \AA$. However, since this assignment is made on the basis of only two reflections, one of which was often not sufficiently intense to even be observed, we are more cautious about this than about the identity of the $H_{I I}$ intermediate.

\section{Lattice parameters and water content}

The lattice parameters of the initial $Q_{I I}^{G}$ and final $Q_{I I}^{D}$ phases are shown in the third and fourth columns of Table I. 
TABLE I. Lattice parameters of $Q_{I I}^{G}$ and $Q_{I I}^{D}$ phase. Lattice parameters are quoted in units of angstroms.

\begin{tabular}{ccccccc}
\hline \hline & $p_{\text {start }}-p_{\text {end }}$ & & \multicolumn{3}{c}{$Q_{I I}^{D}$ first appearance } \\
$T\left({ }^{\circ} \mathrm{C}\right)$ & $($ bar $)$ & Initial $a\left(Q_{I I}^{G}\right)$ & Final $a\left(Q_{I I}^{D}\right)$ & $a\left(Q_{I I}^{G}\right)$ & $a\left(Q_{I I}^{D}\right)$ & Ratio \\
\hline 59.5 & $600-240$ & 184.6 & 112.9 & 183.9 & 115.9 & 1.587 \\
59.5 & $640-230$ & 184.2 & 112.2 & 183.0 & 115.5 & 1.584 \\
59.5 & $670-320$ & 183.5 & 112.6 & 182.6 & 115.7 & 1.578 \\
59.5 & $600-300$ & 181.9 & 111.3 & 180.4 & 113.8 & 1.585 \\
59.5 & $600-360$ & 181.0 & 111.5 & 179.1 & 113.2 & 1.582 \\
57.5 & $630-290$ & 185.6 & 112.2 & 184.1 & 115.7 & 1.591 \\
62.5 & $650-290$ & 181.0 & 109.0 & 180.3 & 113.9 & 1.583 \\
64.6 & $660-300$ & 180.0 & 107.6 & 180.0 & 113.2 & 1.590 \\
\hline \hline
\end{tabular}

The lattice parameter of a $Q_{I I}^{G}$ phase when it is on its own, at the start of a pressure-jump experiment, is geometrically constrained by the phase's water content. In fact, we can estimate the volume fraction of water contained within a $Q_{I I}$ phase from its lattice parameter, from parameters obtained from swelling data obtained at atmospheric pressure [21]. The approach is described in more detail in Ref. [22]. These estimates confirm that $Q_{I I}^{G}$ phases with lattice parameters between 180 and $186 \AA$ have water volume fractions of between 0.48 and 0.495 . The final $Q_{I I}^{D}$ phases have lower water volume fractions, between 0.46 and 0.49 . This suggests that in some cases the final $Q_{I I}^{D}$ phase may coexist with excess water.

It is more interesting to notice the lattice parameters of the two phases at the point where the $Q_{I I}^{D}$ phase first appears, in each pressure-jump experiment, if we want to gain an insight into the mechanism of the transformation. Here, we know that any $Q_{I I}^{D}$ phase present has only just been formed. Moreover, the $Q_{I I}^{G}$ phase at this point is the phase from which it was formed. The lattice parameter values for each phase at this point are also shown in Table I, in the fifth and sixth columns. It is remarkable to note that in every experiment, the ratio $a\left(Q_{I I}^{G}\right) / a\left(Q_{I I}^{D}\right)$ appears to be a constant, and, furthermore, that the value of this ratio is consistent to within \pm 0.01 with the predicted ratio of 1.58 for the lattice parameters of two such TPMSs which are related by the Bonnet transformation. In such cases the $Q_{I I}$ phases may be interconverted by a transformation that conserves topology, as described in Sec. II A. We have described in Sec. I other work where this ratio has been observed in equilibrium studies at phase boundaries, reflecting the fact that two $Q_{I I}$ phases related in this way are expected to have the same bending energy. However, in this case the relationship has been observed in a system very far out of equilibrium, and provides compelling mechanistic evidence that the $Q_{I I}$ intertransformations really do, as we might expect, proceed via a mechanism which conserves topology.

For a given topology, the water contents of $Q_{I I}$ phases increases in the order $\mathrm{G}<\mathrm{D}<\mathrm{P}$. This is a general result arising from the different dimensionless areas of the underlying TPMSs. In this case, water content estimates based on the lattice parameter data shown in Table I suggest that the water volume fraction of the $Q_{I I}^{D}$ phase when it first appears is higher by 0.01 in every pressure-jump experiment than the $Q_{I I}^{G}$ phase from which it forms, taking values between 0.49 and 0.50 . In order to form a phase of a higher water content, the system requires additional water from somewhere. We suggest that the role of the intermediate $H_{I I}$ phase is one of a temporary "water donor;" by forming some of the $H_{I I}$ phase, which has a water volume fraction of around 0.3 , the system releases water, which is required for a topology-conserving transformation from a $Q_{I I}^{G}$ to a $Q_{I I}^{D}$ phase.

All of these observations are to be expected if we bear in mind the considerations outlined at the end of Sec. II. Since it is impossible to conserve both water volume fraction and overall bilayer topology throughout such transformations which are carried out under limited-hydration conditions, the system can at best conserve topology only temporarily, and at a local level, in a process which requires a temporary source of water. In this case, the lattice parameter of the $Q_{I I}^{D}$ phase subsequently decreases, in a process which involves a topological change, in order that the water content of the final $Q_{I I}^{D}$ phase be no higher than the value of the sample overall. We should note the presence of what appears to be an intermediate phase, but may not actually be a mechanistic intermediate, and instead may act as a means of temporarily allowing a surplus or deficit of water. In this case the $H_{I I}$ phase acts as a transient water donor. In another paper [19], we have described how a higher water content $Q_{I I}^{P}$ phase may act as a transient water acceptor. We would expect such features to be observed in other phase transformations carried out under limited hydration conditions.

\section{E. Kinetics}

We assume that the amount of a given phase at any time is proportional to the integrated intensity of one of the x-ray diffraction peaks. This requires that the scattering factor for each phase remain constant. The lattice parameter of both phases does change slightly throughout the phase transitions, but since this is only by a few percent, we assume that we can ignore any effect which this has on the scattering factor of each phase.

Having made this assumption, we can monitor the rate of the phase transition, watching the emergence of the $Q_{I I}^{D}$ phase 

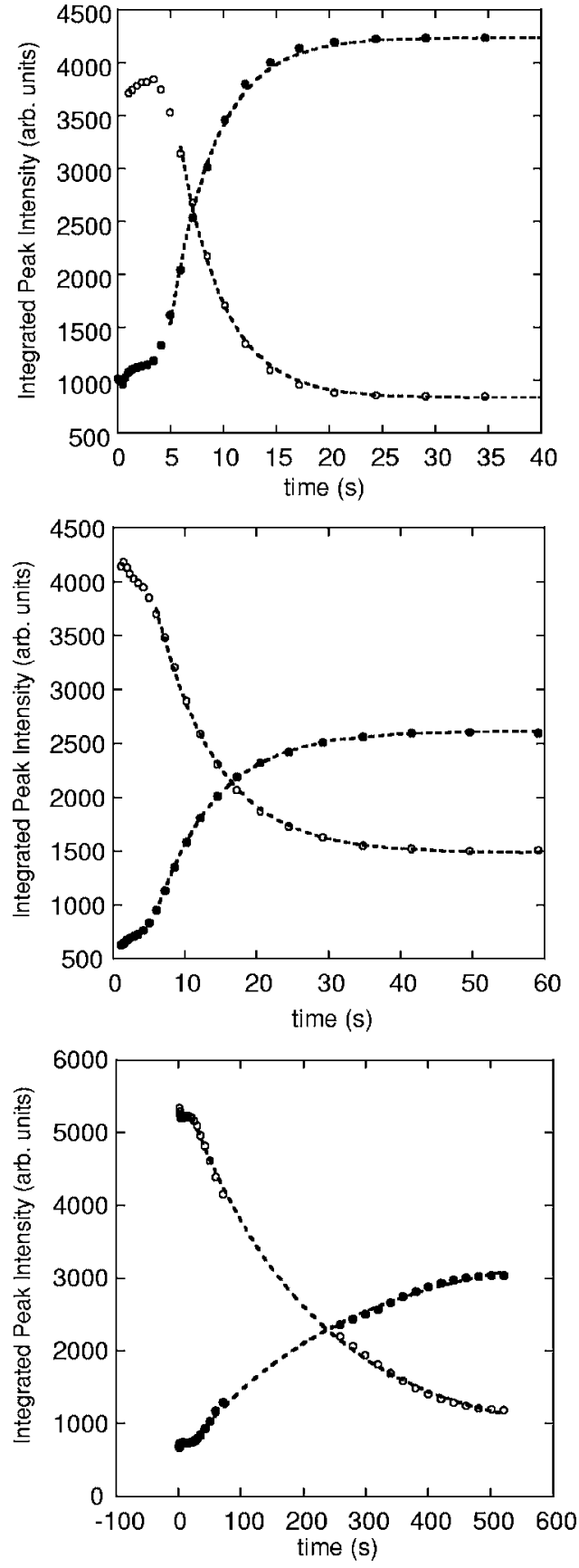

FIG. 8. Pressure jumps carried out using different pressure-jump amplitudes at $T=59.5^{\circ} \mathrm{C}$. Data points showing integrated peak intensities for the emergent $Q_{I I}^{D}$ phase (solid circles) and the disappearing $Q_{I I}^{G}$ phase (hollow circles), and dotted lines showing exponential curve fits. Pressure jumps were carried out from 600 to 240 bar (top), 600 to 300 bar (middle), and 600 to 360 bar (bottom). Between 70 and $258 \mathrm{~s}$ in the third graph, there is a gap in the data, which corresponds to a delay in data acquisition while the acquired images were written on to the hard disk of the image acquisition computer.

at the expense of the $Q_{I I}^{G}$ throughout the transformation. We can then repeat the pressure jump under different conditions, and watch how the transition kinetics depend on these conditions.
TABLE II. Effects of pressure-jump amplitude on rate constant. Pressure jumps were carried out at $T=59.5^{\circ} \mathrm{C}$, from pressure $p_{\text {start }}$ to pressure $p_{\text {end }}$. The first was carried out at DESY, in an experiment described elsewhere [19], the others at the ESRF.

\begin{tabular}{cccc}
\hline \hline $\begin{array}{c}p_{\text {start }} \\
\text { (bar) }\end{array}$ & $\begin{array}{c}p_{\text {end }} \\
(\mathrm{bar})\end{array}$ & $\begin{array}{c}k\left(\mathrm{~s}^{-1}\right) \\
Q_{I I}^{G}\end{array}$ & $Q_{I I}^{D}$ \\
\hline 600 & 190 & $>1$ & $>1$ \\
600 & 240 & $0.237 \pm 0.009$ & $0.241 \pm 0.008$ \\
600 & 300 & $0.118 \pm 0.003$ & $0.120 \pm 0.002$ \\
600 & 360 & $0.0049 \pm 0.0002$ & $0.0038 \pm 0.0002$ \\
\hline \hline
\end{tabular}

\section{Effects of pressure-jump amplitude}

Figure 8 shows the changing peak intensities as functions of time for three pressure jumps carried out at the same temperature, and starting at the same pressure, but ending at progressively higher pressures, thus decreasing the pressurejump amplitude each time. In general, all three pressure jumps show similar features. Immediately following the pressure jump, there is a delay of around 5-20 s, when very little seems to change.

What exactly is happening during this delay is unclear. We can certainly rule out some artifact of the apparatus because the duration of the delay does vary with the time scale of the phase transition. It is possible that some change is occurring at certain defects, which will then serve as nucleation sites for the transformation. These sites represent such a small proportion of the sample as a whole that they may well not affect the overall diffraction pattern.

After this, the $Q_{I I}^{G}$ disappears and the $Q_{I I}^{D}$ phase emerges, as we would expect.

We have fitted the data after the initial delay with an exponential curve, of the form

$$
I_{t}=I_{\infty}+A e^{-k t} .
$$

Here, $I_{t}$ represents the integrated intensity of the peak at time $t$, and the exponential decay is parametrized by the three constants $I_{\infty}, A$, and $k$. The value of $A$ can be positive or negative, respectively reflecting cases where the signal intensity decreases or increases during the transformation. $I_{\infty}$ represents the final peak intensity, estimated by extrapolating the exponential curve to $t=\infty$. The first-order rate constant is represented by $k$.

The exponential curve fits are also shown in Fig. 8. In general this fit gives a good description of the data, although there are some small but systematic deviations away from exponential behavior. However, we are using exponential fits merely as a way of obtaining rate constants to facilitate quantitative comparison, and are not attaching any mechanistic significance to the exponential curve shape itself. A deviation from exponential behavior should therefore not concern us.

The first-order rate constants for both the appearance of the $Q_{I I}^{D}$ phase and the disappearance of the $Q_{I I}^{G}$ phase are summarized in Table II. We also include the very fast phase transition that we have described in Ref. [19]. Two features become apparent.

(1) There is a very good agreement between the rate con- 

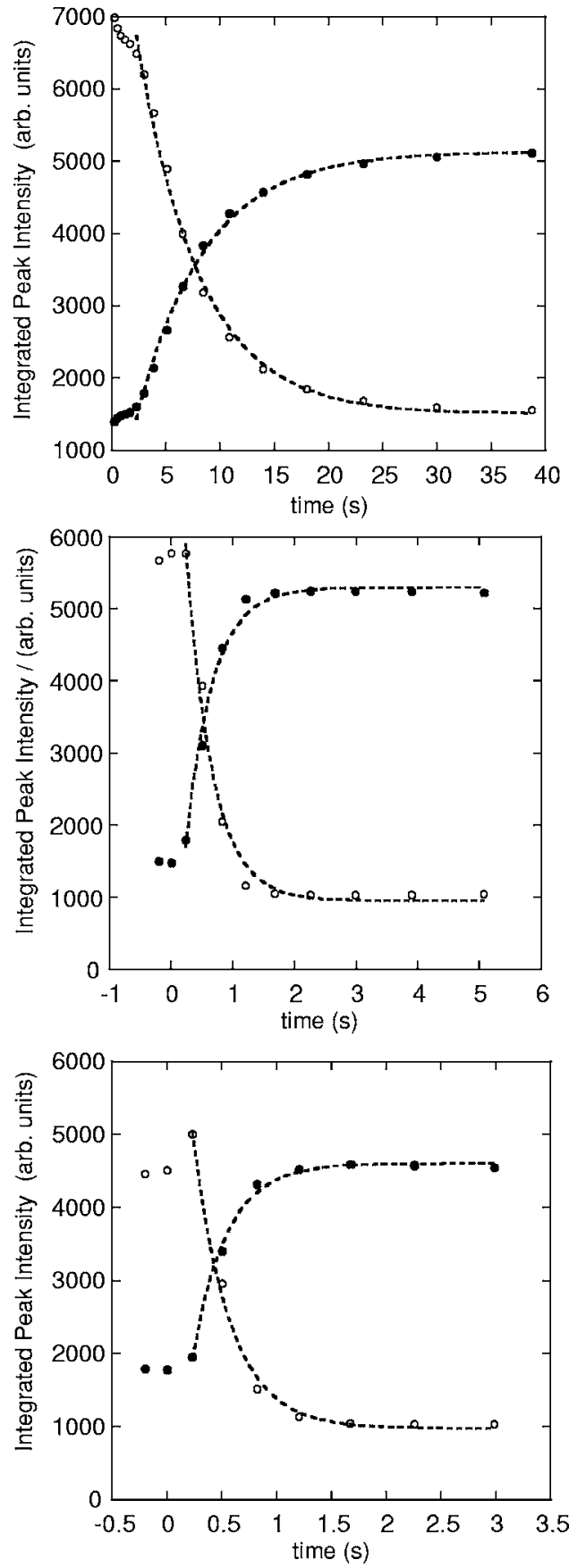

FIG. 9. Pressure jumps carried out at different temperatures. Data points showing integrated peak intensities for the emergent $Q_{I I}^{D}$ phase (solid circles) and the disappearing $Q_{I I}^{G}$ phase (hollow circles), and dotted lines showing exponential curve fits. The experimental conditions employed were $57.5^{\circ} \mathrm{C}, 630-290$ bar (top); $62.5^{\circ} \mathrm{C}, 650-290$ bar (middle); and $64.6^{\circ} \mathrm{C}, 660-300$ bar (bottom).

stants for the appearance of the $Q_{I I}^{D}$ phase and the disappearance of the $Q_{I I}^{G}$ phase, for a given pressure jump. This is, of course, as we would expect.

(2) There is a clear increase in the overall rate of the transformation with the amplitude of the pressure jump employed.

This last result is in agreement with data obtained by Erbes and co-workers [20], who suggested that an increase in
TABLE III. Effects of temperature on rate constant. Pressure jumps were carried out at temperature $T$, from pressure $p_{\text {start }}$ to pressure $p_{\text {end }}$. Rate constants are shown for both the disappearance of $Q_{I I}^{G}$ and the appearance of $Q_{I I}^{D}$.

\begin{tabular}{ccccc}
\hline \hline $\begin{array}{c}p_{\text {start }} \\
(\mathrm{bar})\end{array}$ & $\begin{array}{c}p_{\text {end }} \\
(\mathrm{bar})\end{array}$ & $\begin{array}{c}T \\
\left({ }^{\circ} \mathrm{C}\right)\end{array}$ & $\begin{array}{c}k\left(\mathrm{~s}^{-1}\right) \\
Q_{I I}^{G}\end{array}$ & $Q_{I I}^{D}$ \\
\hline 630 & 290 & 57.5 & $0.17 \pm 0.01$ & $0.16 \pm 0.01$ \\
650 & 290 & 62.5 & $2.4 \pm 0.3$ & $2.3 \pm 0.3$ \\
660 & 300 & 64.6 & $3.0 \pm 0.3$ & $3.3 \pm 0.3$ \\
\hline \hline
\end{tabular}

pressure-jump amplitude causes an increase in the chemical potential difference between the phases, and therefore in the thermodynamic "driving force" for the transformation.

\section{Temperature}

A further set of pressure-jump experiments was carried out using approximately the same start and end pressures, but at different temperatures. The data sets from these experiments are shown in Fig. 9. Again, these can be fitted with exponential curves, and the first-order rate constants that these yield are summarized in Table III.

The data show a clear increase in rate with temperature.

This trend may be explained using a standard Arrheniustype approach. A plot of $\ln k$ against $1 / T$ suggests an activation energy of around $50 \pm 10 \mathrm{~kJ} \mathrm{~mol}^{-1}$. This is shown in Fig. 10. The fit is not a very good one, and furthermore it is unclear how this value should be interpreted, and in particular, to which species the unit of $\mathrm{kJ} \mathrm{mol}^{-1}$ refers. Unlike cases involving classical solution-phase kinetics, the entity undergoing change is not a single lipid molecule or complex, but a domain consisting of thousands of molecules. An interesting interpretation has been offered by Schoppe et al. [23], who suggest the concept of a "cooperative unit," which they define as "the number of molecules that undergo the transition cooperatively in a synchronized two-state manner." Thus it is possible that a calculated activation energy is the energy per mole of these cooperative units. However, the issue is far from being resolved.

Moreover, there is an alternative explanation for the observed increase in rate with temperature, which relates to the effects of pressure-jump amplitude described in Sec. IV E 1. Erbes et al. [20] suggest that the rate of a phase transition depends more specifically on the distance of the final pres-

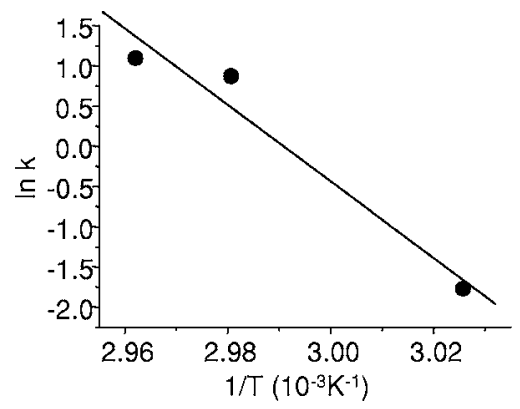

FIG. 10. Arrhenius plot showing data from Table III. 
sure $p_{\text {end }}$ from the pressure of the phase transition boundary, $p_{t r}$. In the experiments described here, although the initial and final pressures are kept constant, the transition boundary moves to higher and higher values as we increase the temperature (see Fig. 5). The value of $\left(p_{t r}-p_{\text {end }}\right)$ therefore increases. We suggest that it may be this, rather than the effect of an "activation energy," which causes the observed increase in rate with temperature.

These two explanations may easily be discriminated between when data become available on the effect of temperature on the rate of the reverse $Q_{I I}^{D} Q_{I I}^{G}$ transformation, keeping the initial and final pressures constant. Since the transition in this case would be induced by a jump to higher pressures, the increase in transition pressure with temperature would therefore cause a decrease in the size of ( $p_{\text {end }}$ $-p_{t r}$, and, we suggest, a decrease in rate. On the other hand, an argument based on activation energy would predict that the rate of phase transitions in both directions should increase with temperature. Arguments based on activation energy therefore suggest that the $Q_{I I}^{D}$ to $Q_{I I}^{G}$ transition should proceed more rapidly at higher temperatures, while arguments based on the thermodynamic driving force represented by the size of $\left(p_{t r}-p_{\text {end }}\right)$ suggest the opposite.

\section{SUMMARY AND CONCLUSIONS}

We have described, and shown graphically, topologyconserving mechanisms that interconvert the $Q_{I I}^{P}, Q_{I I}^{D}$, and $Q_{I I}^{G}$ mesophases, based on the work of others. If the pathway shown does indeed preserve bilayer topology, the lattice parameters of the two mesophases will have a ratio of $a(G) / a(D)=1.576$ or $a(P) / a(D)=1.279$. The description also predicts a relative rotation of $60^{\circ}$ about a common $(1,1,1)$ axis for the $Q_{I I}^{P} Q_{I I}^{D}$ transformation, and of $45^{\circ}$ about a common $(0,0,1)$ axis for $Q_{I I}^{D} Q_{I I}^{G}$. In addition, we would expect a change in the aspect ratio of a single crystallite by a factor of 2 in the $(1,1,1)$ direction for $Q_{I I}^{P} Q_{I I}^{D}$, and by a factor of $\sqrt{2}$ in the $(0,0,1)$ direction for $Q_{I I}^{D} Q_{I I}^{G}$

We have presented time-resolved x-ray diffraction data for the $Q_{I I}^{G} Q_{I I}^{D}$ transformation induced by a pressure jump. These show that, after an initial delay, both the disappearance of the $Q_{I I}^{G}$ mesophase and the appearance of the $Q_{I I}^{D}$ follow approximately exponential kinetics, with the same first-order rate constant for each pressure-jump experiment. The value of this rate constant varies from the order of seconds to minutes, increasing with both pressure-jump amplitude and temperature.

The data show that in each case, the ratio of $a(G) / a(D)$ at the stage where the $Q_{I I}^{D}$ first appears is 1.58 . Our calculations suggest that the water content of the new $Q_{I I}^{D}$ phase is slightly higher than that of the $Q_{I I}^{G}$ phase from which it formed. We also observe a transient $H_{I I}$ phase, which we suggest compensates for this change in lipid:water ratio. All of this is consistent with a mechanism that conserves bilayer topology. In addition, we observe another very short-lived signal at $d$ $=53-55 \AA$. At the moment, the role of this intermediate is unclear.

Since our data were obtained from a powder-averaged polydomain sample, we cannot test the predicted changes in crystallite orientation or aspect ratio. To address both of these issues will require data from single crystallites. We expect that, when such data do become available, they will unambiguously confirm or disprove the described transition mechanism.

\section{ACKNOWLEDGMENTS}

A.M.S. acknowledges support from EPSRC EPSRC. We would like to thank S. Hyde for useful discussions.

\section{APPENDIX: NUMERICAL DESCRIPTIONS OF TRANSFORMATIONS OF SKELETAL GRAPHS AND BILAYER MIDPLANE SURFACES}

In this appendix we provide numerical expressions that can be used to generate both the surfaces and the skeletal graphs shown in Figs. 2 and 3. These represent, respectively, the approximate positions of the middle of the bilayer and the centers of the water channels. We have considered the transformations described in Sec. II to consist of three processes; a rearrangement within the repeat cell, a change in the shape of the repeat cell, and a rescaling, in order to conserve bilayer area. We can quantify any intermediate structure generated by the first two processes, using two parameters, which we will denote $u$ and $v$. These represent the distortion of the repeat cell and the rearrangement within the repeat cell, respectively.

The approximate bilayer midplane positions are generated using periodic nodal surfaces (PNSs) [24,25]. The PNSs for the three $Q_{I I}$ phases are given by the solutions of the following equations:

$$
\begin{aligned}
F^{P}= & \cos (2 \pi x)+\cos (2 \pi y)+\cos (2 \pi z)=0, \\
F^{D}= & \cos (2 \pi x) \cos (2 \pi y) \cos (2 \pi z) \\
& -\sin (2 \pi x) \sin (2 \pi y) \sin (2 \pi z)=0, \\
F^{G}= & \sin (2 \pi x) \cos (2 \pi y)+\sin (2 \pi y) \cos (2 \pi z) \\
+ & \sin (2 \pi z) \cos (2 \pi x)=0 .
\end{aligned}
$$

These are very similar, although not identical, to the P, D, and G TPMSs.

The skeletal graphs are assumed to be networks of straight channels connecting three- four- or six-way junctions. Such constructions give a good representation of the topology and general shape of the water channels. However, unlike medial graph constructions, they do not necessarily describe the exact position of the channels' centers. The calculation of such a position is not trivial, and is the subject of much ongoing research $[26,15]$. With this in mind, we do not attach too much significance to the exact shapes of the two representations of intermediate structures in mesophase rearrangement within the unit cell. For this reason, we use two different letters to denote the rearrangement, $v$ for the transformation of the bilayer midplane surface, and $v^{\prime}$ for that of the skeletal graph, and we note that there may not be an exact correlation between the two. 
TABLE IV. Coordinates used in the construction of a generalized skeletal graph representing the $Q_{I I}^{G}$ $-Q_{I I}^{D}$ transformation

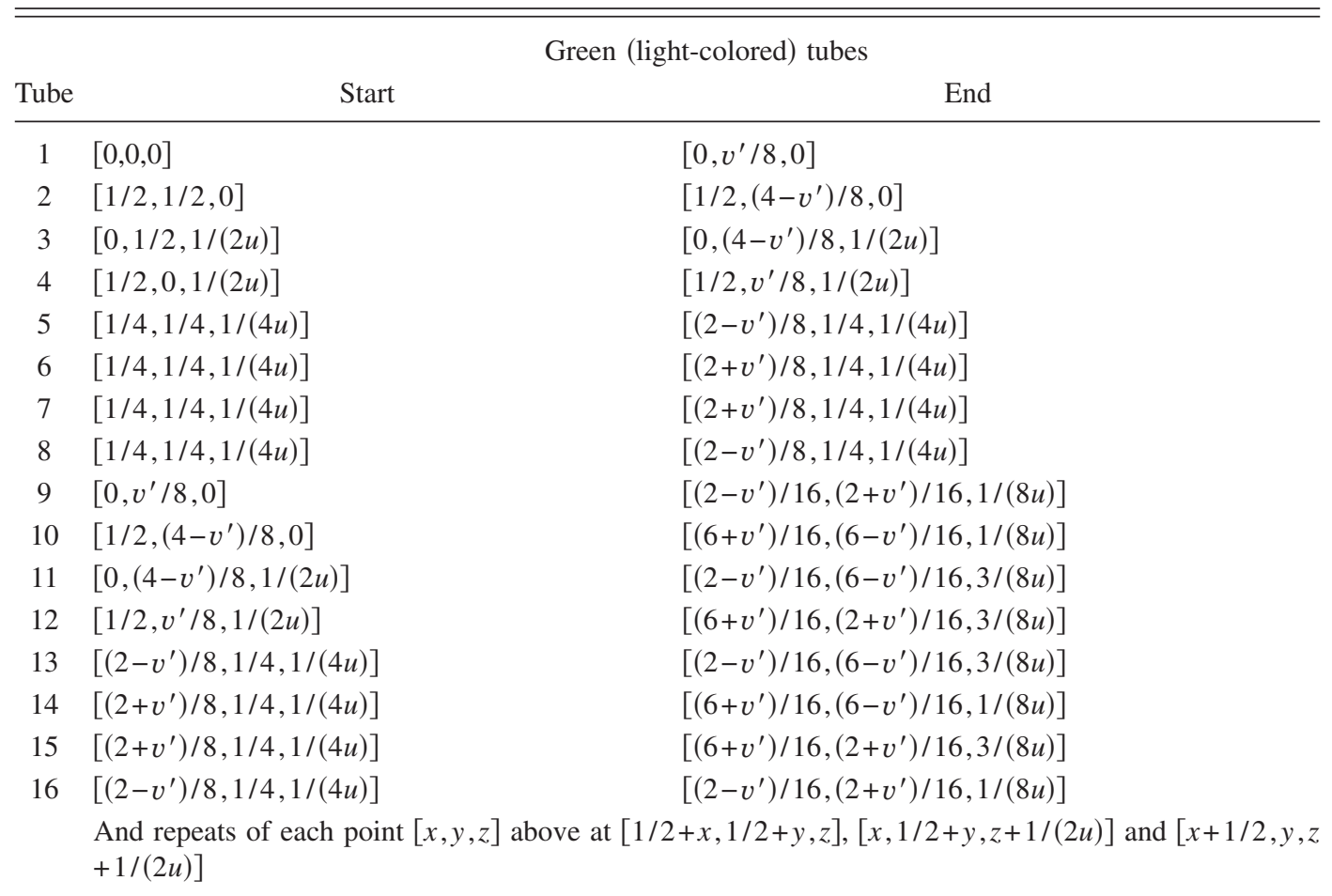

Red (dark-colored) tubes

\begin{tabular}{cll} 
Tube & \multicolumn{1}{c}{ Start } & \multicolumn{1}{c}{ End } \\
\hline 1 & {$[1 / 2,0,0]$} & {$\left[\left(4+v^{\prime}\right) / 8,0,0\right]$} \\
2 & {$[1,1 / 2,0]$} & {$\left[1-v^{\prime} / 8,1 / 2,0\right]$} \\
3 & {$[1 / 2,1 / 2,1 /(2 u)]$} & {$\left[\left(4+v^{\prime}\right) / 8,1 / 2,1 /(2 u)\right]$} \\
4 & {$[1,0,1 /(2 u)]$} & {$\left[1-v^{\prime} / 8,0,1 /(2 u)\right]$} \\
5 & {$[3 / 4,1 / 4,1 /(4 u)]$} & {$\left[3 / 4,\left(2+v^{\prime}\right) / 8,1 /(4 u)\right]$} \\
6 & {$[3 / 4,1 / 4,1 /(4 u)]$} & {$\left[3 / 4,\left(2+v^{\prime}\right) / 8,1 /(4 u)\right]$} \\
7 & {$[3 / 4,1 / 4,1 /(4 u)]$} & {$\left[3 / 4,\left(2-v^{\prime}\right) / 8,1 /(4 u)\right]$} \\
8 & {$[3 / 4,1 / 4,1 /(4 u)]$} & {$\left[\left(10+v^{\prime}\right) / 16,\left(2-v^{\prime}\right) / 16,1 /(8 u)\right]$} \\
9 & {$\left[\left(4+v^{\prime}\right) / 8,0,0\right]$} & {$\left[\left(14-v^{\prime}\right) / 16,\left(6+v^{\prime}\right) / 16,1 /(8 u)\right]$} \\
10 & {$\left[1-v^{\prime} / 8,1 / 2,0\right]$} & {$\left[\left(10+v^{\prime}\right) / 16,\left(6+v^{\prime}\right) / 16,3 /(8 u)\right]$} \\
11 & {$\left[\left(4+v^{\prime}\right) / 8,1 / 2,1 /(2 u)\right]$} & {$\left[\left(14-v^{\prime}\right) / 16,\left(2-v^{\prime}\right) / 16,3 /(8 u)\right]$} \\
12 & {$\left[1-v^{\prime} / 8,0,1 /(2 u)\right]$} & {$\left[\left(10+v^{\prime}\right) / 16,\left(6+v^{\prime}\right) / 16,3 /(8 u)\right]$} \\
13 & {$\left[3 / 4,\left(2+v^{\prime}\right) / 8,1 /(4 u)\right]$} & {$\left[\left(14-v^{\prime}\right) / 16,\left(2-v^{\prime}\right) / 16,3 /(8 u)\right]$} \\
14 & {$\left[3 / 4,\left(2-v^{\prime}\right) / 8,1 /(4 u)\right]$} & {$\left[\left(14-v^{\prime}\right) / 16,\left(6+v^{\prime}\right) / 16,1 /(8 u)\right]$} \\
15 & {$\left[3 / 4,\left(2+v^{\prime}\right) / 8,1 /(4 u)\right]$} & {$\left[\left(10+v^{\prime}\right) / 16,\left(2-v^{\prime}\right) / 16,1 /(8 u)\right]$} \\
16 & {$\left[3 / 4,\left(2-v^{\prime}\right) / 8,1 /(4 u)\right]$} & \\
& And repeats of each point $[x, y, z]$ above at $[x-1 / 2, y+1 / 2, z],[x, y+1 / 2, z+1 /(2 u)]$ and $[x-1 / 2, y, z$ \\
& $+1 /(2 u)]$ & \\
\hline \hline & & \\
\hline
\end{tabular}

\section{The $Q_{I I}^{D}-Q_{I I}^{G}$ transformation}

We represent the extent of the tetragonal squashing of the repeat cell defined in Sec. II A by the parameter $u$, which takes values from 1 for the $\mathrm{D}$ minimal surface (where the repeat cell is a cube of dimension $1 \times 1 \times 1)$, and $\sqrt{2}$ for the $\mathrm{G}$ minimal surface [where the repeat cell dimensions are now $1 \times 1 \times(1 / \sqrt{2})]$. For a given value of $u$, the two limiting surfaces (which, as a result of the tetragonal distortion of the repeat cell, are no longer necessarily minimal surfaces) are tetragonally distorted $\mathrm{D}$ and $\mathrm{G}$ surfaces. These can be represented by limiting periodic nodal surfaces.

One limiting periodic nodal surface resembles a D TPMS that has been translated through $(1 / 4,-1 / 4,1 / 4)$ and squashed by a factor $u$ along the $z$ axis. Equation (A2) becomes 
TABLE V. Coordinates used in the construction of a generalized skeletal graph representing the $Q_{I I}^{P}$ - $Q_{I I}^{D}$ transformation

\begin{tabular}{llc}
\hline \hline \multicolumn{1}{c}{ Tube } & Start & Green (light-colored) tubes \\
& & $\left.\left[u\left(4-v^{\prime}\right) / 8, u\left(4-v^{\prime}\right) / 8, u\left(4-v^{\prime}\right)\right) / 8\right]$ \\
1 & {$[u / 2, u / 2, u / 2]$} & $\left.\left[u\left(4+v^{\prime}\right) / 8, u\left(4+v^{\prime}\right) / 8, u\left(4+v^{\prime}\right)\right) / 8\right]$ \\
2 & {$[u / 2, u / 2, u / 2]$} & {$[(1+2 u) / 6,(1+2 u) / 6,(-1+u) / 3]$} \\
3 & {$\left[u\left(4-v^{\prime}\right) / 8, u\left(4-v^{\prime}\right) / 8, u\left(4-v^{\prime}\right) / 8\right]$} & {$[(1+2 u) / 6,(-1+u) / 3,(1+2 u) / 6]$} \\
4 & {$\left[u\left(4-v^{\prime}\right) / 8, u\left(4-v^{\prime}\right) / 8, u\left(4-v^{\prime}\right) / 8\right]$} & {$[(-1+u) / 3,(1+2 u) / 6,(1+2 u) / 6]$} \\
5 & {$\left[u\left(4-v^{\prime}\right) / 8, u\left(4-v^{\prime}\right) / 8, u\left(4-v^{\prime}\right) / 8\right]$} & {$[(-1+4 u) / 6,(-1+4 u) / 6,(1+2 u) / 3]$} \\
6 & {$\left[u\left(4+v^{\prime}\right) / 8, u\left(4+v^{\prime}\right) / 8, u\left(4+v^{\prime}\right) / 8\right]$} & {$[(-1+4 u) / 6,(1+2 u) / 3,(-1+4 u) / 6]$} \\
7 & {$\left[u\left(4+v^{\prime}\right) / 8, u\left(4+v^{\prime}\right) / 8, u\left(4+v^{\prime}\right) / 8\right]$} & $[(1+2 u) / 3,(-1+4 u) / 6,-1+4 u) / 6]$
\end{tabular}

Red (dark-colored) tubes

Tube Start End

$$
\begin{aligned}
& 1 \quad[0,0,0] \\
& 2 \quad[u, u, u] \\
& 3 \quad\left[\left(u v^{\prime}\right) / 8,\left(u v^{\prime}\right) / 8,\left(u v^{\prime}\right) / 8\right] \\
& 4 \quad\left[\left(u v^{\prime}\right) / 8,\left(u v^{\prime}\right) / 8,\left(u v^{\prime}\right) / 8\right] \\
& 5 \quad\left[\left(u v^{\prime}\right) / 8,\left(u v^{\prime}\right) / 8,\left(u v^{\prime}\right) / 8\right] \\
& 6 \quad\left[u-\left(u v^{\prime}\right) / 8, u-\left(u v^{\prime}\right) / 8, u-\left(u v^{\prime}\right) / 8\right] \\
& 7 \quad\left[u-\left(u v^{\prime}\right) / 8, u-\left(u v^{\prime}\right) / 8, u-\left(u v^{\prime}\right) / 8\right] \\
& 8 \quad\left[u-\left(u v^{\prime}\right) / 8, u-\left(u v^{\prime}\right) / 8, u-\left(u v^{\prime}\right) / 8\right] \\
& F_{u}^{D}(x, y, z)=\cos \left[2 \pi\left(x+\frac{1}{4}\right)\right] \cos \left[2 \pi\left(y-\frac{1}{4}\right)\right] \\
& \times \cos \left[2 \pi\left(u z+\frac{1}{4}\right)\right]-\sin \left[2 \pi\left(x+\frac{1}{4}\right)\right] \\
& \times \sin \left[2 \pi\left(y-\frac{1}{4}\right)\right] \sin \left[2 \pi\left(u z+\frac{1}{4}\right)\right]=0 .
\end{aligned}
$$

The other periodic nodal surface resembles a G TPMS that has been scaled down by a factor of $\sqrt{2}$, rotated by $45^{\circ}$ about the $z$ axis, stretched along the $z$ axis by a factor of $\sqrt{2} / u$, and translated to give

$$
\begin{aligned}
F_{u}^{G}(x, y, z)= & \sin [2 \pi(x-y)] \cos \left[2 \pi\left(x+y+\frac{1}{4}\right)\right] \\
& +\sin \left[2 \pi\left(x+y+\frac{1}{4}\right)\right] \cos \left[2 \pi\left(u z+\frac{1}{8}\right)\right] \\
& +\sin \left[2 \pi\left(u z+\frac{1}{8}\right)\right] \cos [2 \pi(x-y)]=0
\end{aligned}
$$

We can approximate intermediate surfaces representing the rearrangement of water channels within the unit cell by taking a linear combination of the two expressions in the two previous equations. The extent of the rearrangement is quantified by the parameter $v$, which takes values from 0 (limiting case derived from distorted D) to 1 (limiting case derived from distorted $G$ ). We can now define any intermediate surface in terms of the two parameters $u$ and $v$. The equation for this generalized surface is

$$
\begin{aligned}
& {\left[\left(u v^{\prime}\right) / 8,\left(u v^{\prime}\right) / 8,\left(u v^{\prime}\right) / 8\right]} \\
& {\left[u-\left(u v^{\prime}\right) / 8, u-\left(u v^{\prime}\right) / 8, u-\left(u v^{\prime}\right) / 8\right]} \\
& {[(2+u) / 6,(-1+u) / 6,(-1+u) / 6]} \\
& {[(-1+u) / 6,(-1+u) / 6,(2+u) / 6]} \\
& {[(-1+u) / 6,(2+u) / 6,(-1+u) / 6]} \\
& {[(-2+5 u) / 6,(1+5 u) / 6,(1+5 u) / 6]} \\
& {[(1+5 u) / 6,(1+5 u) / 6,(-2+5 u) / 6]} \\
& {[(1+5 u) / 6,(-2+5 u) / 6,(1+5 u) / 6]}
\end{aligned}
$$

$$
F_{u, v}^{D-G}(x, y, z)=v F_{u}^{G}(x, y, z)+(1-v) F_{u}^{D}(x, y, z)=0,
$$

where, in its expanded form,

$$
\begin{aligned}
F_{u, v}^{D-G}(x, y, z)= & v\left\{\sin [2 \pi(x-y)] \cos \left[2 \pi\left(x+y+\frac{1}{4}\right)\right]\right. \\
& +\sin \left[2 \pi\left(x+y+\frac{1}{4}\right)\right] \cos \left[2 \pi\left(u z+\frac{1}{8}\right)\right] \\
& \left.+\sin \left[2 \pi\left(u z+\frac{1}{8}\right)\right] \cos [2 \pi(x-y)]\right\}+(1-v) \\
& \times\left\{\cos \left[2 \pi\left(x+\frac{1}{4}\right)\right] \cos \left[2 \pi\left(y-\frac{1}{4}\right)\right]\right. \\
& \times \cos \left[2 \pi\left(u z+\frac{1}{4}\right)\right]-\sin \left[2 \pi\left(x+\frac{1}{4}\right)\right] \\
& \left.\times \sin \left[2 \pi\left(y-\frac{1}{4}\right)\right] \sin \left[2 \pi\left(u z+\frac{1}{4}\right)\right]\right\} .
\end{aligned}
$$

The limiting cases for $(u=1, v=0)$ and $(u=\sqrt{2}, v=1)$ correspond to the periodic nodal surfaces for undistorted D and $G$, respectively.

We can generate the skeletal graphs representing the water channel networks shown in Fig. 2 by repeated use of the 'surf' function in MATLAB to generate a series of tubes, which connect to form the skeletal graphs. The coordinates of the ends of the dark (red) and light (green) tubes for one quadrant of the structure shown in Fig. 2 are listed in Table IV. These coordinates are functions of the parameters $u$ and $v^{\prime}$ which again represent the distortion of the repeat cell, and 
the rearrangement within the repeat cell, so that the coordinates obtained for $\left(u=1, v^{\prime}=0\right)$ and $\left(u=\sqrt{2}, v^{\prime}=1\right)$ generate the skeletal graphs for undistorted D and G, respectively.

\section{The $Q_{I I}^{P}-Q_{I I}^{D}$ transformation}

We represent the extent of stretching of the repeat cell defined in Sec. II B in the $(1,1,1)$ direction by the parameter $u$, which now takes values from 1 for the $\mathrm{P}$ minimal surface (where the repeat cell is a cube of dimension $1 \times 1 \times 1$ ), to 2 for the D minimal surface [where the repeat cell is a rhombohedron whose main triple symmetry axis runs from $(0,0,0)$ to $(2,2,2)]$. For a given value of $u$, the two limiting surfaces are now rhombohedrally distorted $\mathrm{P}$ and $\mathrm{D}$ surfaces. These can be represented by limiting periodic nodal surfaces.

One limiting periodic nodal surface resembles a P TPMS that has been rhombohedrally stretched by a factor $u$ along the $(1,1,1)$ axis. The equation for this, obtained from Eq. (A1), is

$$
\begin{aligned}
F_{u}^{P}(x, y, z)= & \cos \left\{\frac{2 \pi}{3}\left[\left(\frac{1}{u}+2\right) x+\left(\frac{1}{u}-1\right) y+\left(\frac{1}{u}-1\right) z\right]\right\} \\
& +\cos \left\{\frac{2 \pi}{3}\left[\left(\frac{1}{u}-1\right) x+\left(\frac{1}{u}+2\right) y+\left(\frac{1}{u}-1\right) z\right]\right\} \\
& +\cos \left\{\frac{2 \pi}{3}\left[\left(\frac{1}{u}-1\right) x+\left(\frac{1}{u}-1\right) y+\left(\frac{1}{u}+2\right) z\right]\right\} \\
= & 0 .
\end{aligned}
$$

The other periodic nodal surface resembles a D TPMS [Eq. (A2)] that has been rotated by $60^{\circ}$ about the $(1,1,1)$ axis, squashed by a factor of $(2 / u)$ in the $(1,1,1)$ direction, and translated to give

$$
\begin{aligned}
F_{u}^{D}(x, y, z)= & \cos \left\{-\frac{\pi}{4}+\frac{\pi}{3}\left[\left(\frac{2}{u}+1\right) x+\left(\frac{2}{u}-2\right) y+\left(\frac{2}{u}+1\right) z\right]\right\} \cos \left\{-\frac{\pi}{4}+\frac{\pi}{3}\left[\left(\frac{2}{u}+1\right) x+\left(\frac{2}{u}+1\right) y+\left(\frac{2}{u}-2\right) z\right]\right\} \\
& \times \cos \left\{-\frac{\pi}{4}+\frac{\pi}{3}\left[\left(\frac{2}{u}-2\right) x+\left(\frac{2}{u}+1\right) y+\left(\frac{2}{u}+1\right) z\right]\right\}-\sin \left\{-\frac{\pi}{4}+\frac{\pi}{3}\left[\left(\frac{2}{u}+1\right) x+\left(\frac{2}{u}-2\right) y+\left(\frac{2}{u}+1\right) z\right]\right\} \\
& \times \sin \left\{-\frac{\pi}{4}+\frac{\pi}{3}\left[\left(\frac{2}{u}+1\right) x+\left(\frac{2}{u}+1\right) y+\left(\frac{2}{u}-2\right) z\right]\right\} \sin \left\{-\frac{\pi}{4}+\frac{\pi}{3}\left[\left(\frac{2}{u}-2\right) x+\left(\frac{2}{u}+1\right) y+\left(\frac{2}{u}+1\right) z\right]\right\}=0 .
\end{aligned}
$$

We again approximate intermediate surfaces representing the rearrangement of water channels within the unit cell by taking a linear combination of the two previous two expressions, with the extent of the rearrangement quantified by the parameter $v$. The limiting cases where $v$ has values of 0 and 1 now represent, respectively, the limiting case derived from distorted $\mathrm{P}$ and the limiting case derived from distorted $\mathrm{D}$. The equation for the generalized surface in terms of the two parameters $u$ and $v$ is

$$
F_{u, v}^{P-D}(x, y, z)=v F_{u}^{D}(x, y, z)+(1-v) F_{u}^{P}(x, y, z)=0,
$$

where, in its expanded form,

$$
\begin{aligned}
F_{u, v}^{P-D}(x, y, z)= & v\left(\cos \left\{-\frac{\pi}{4}+\frac{\pi}{3}\left[\left(\frac{2}{u}+1\right) x+\left(\frac{2}{u}-2\right) y+\left(\frac{2}{u}+1\right) z\right]\right\} \cos \left\{-\frac{\pi}{4}+\frac{\pi}{3}\left[\left(\frac{2}{u}+1\right) x+\left(\frac{2}{u}+1\right) y+\left(\frac{2}{u}-2\right) z\right]\right\}\right. \\
& \times \cos \left\{-\frac{\pi}{4}+\frac{\pi}{3}\left[\left(\frac{2}{u}-2\right) x+\left(\frac{2}{u}+1\right) y+\left(\frac{2}{u}+1\right) z\right]\right\}-\sin \left\{-\frac{\pi}{4}+\frac{\pi}{3}\left[\left(\frac{2}{u}+1\right) x+\left(\frac{2}{u}-2\right) y+\left(\frac{2}{u}+1\right) z\right]\right\} \\
& \left.\times \sin \left\{-\frac{\pi}{4}+\frac{\pi}{3}\left[\left(\frac{2}{u}+1\right) x+\left(\frac{2}{u}+1\right) y+\left(\frac{2}{u}-2\right) z\right]\right\} \sin \left\{-\frac{\pi}{4}+\frac{\pi}{3}\left[\left(\frac{2}{u}-2\right) x+\left(\frac{2}{u}+1\right) y+\left(\frac{2}{u}+1\right) z\right]\right\}\right) \\
& +(1-v)\left(\cos \left\{\frac{2 \pi}{3}\left[\left(\frac{1}{u}+2\right) x+\left(\frac{1}{u}-1\right) y+\left(\frac{1}{u}-1\right) z\right]\right\}+\cos \left\{\frac{2 \pi}{3}\left[\left(\frac{1}{u}-1\right) x+\left(\frac{1}{u}+2\right) y+\left(\frac{1}{u}-1\right) z\right]\right\}\right. \\
& \left.+\cos \left\{\frac{2 \pi}{3}\left[\left(\frac{1}{u}-1\right) x+\left(\frac{1}{u}-1\right) y+\left(\frac{1}{u}+2\right) z\right]\right\}\right)=0
\end{aligned}
$$

The limiting cases for $(u=1, v=0)$ and $(u=2, v=1)$ correspond to the periodic nodal surfaces for undistorted $\mathrm{P}$ and $\mathrm{D}$, respectively.

Again, we generate the skeletal graphs representing the water channel networks shown in Fig. 3 by generating another series of tubes. The coordinates of the ends of these tubes are listed in Table $\mathrm{V}$, and the values obtained for $\left(u=1, v^{\prime}=0\right)$ and $\left(u=2, v^{\prime}=1\right)$ correspond to the skeletal graphs of the undistorted $\mathrm{P}$ and $\mathrm{D}$ structures. 
[1] S. T. Hyde, S. Andersson, B. Ericsson, and K. Larsson, Z. Kristallogr. 168, 213 (1984).

[2] A. L. Mackay, Nature (London) 314, 604 (1985).

[3] V. Mariani, P. Luzzati, and H. Delacroix, J. Mol. Biol. 204, 165 (1988).

[4] M. Caffrey, Curr. Opin. Struct. Biol. 10, 486 (2000).

[5] Calum J. Drummond and Celesta Fong, Curr. Opin. Colloid Interface Sci. 4, 449 (2000).

[6] National Academy of Sciences report, 1996, http:// www.nap.edu/readingroom/books/bmm/

[7] S. Andersson, S. T. Hyde, K. Larsson, and S. Lidin, Chem. Rev. (Washington, D.C.) 88, 221 (1988).

[8] J. M. Seddon and R. H. Templer, Philos. Trans. R. Soc. London, Ser. A 344, 377 (1993).

[9] R. H. Templer, Curr. Opin. Colloid Interface Sci. 3, 255 (1998).

[10] S. T. Hyde and S. Andersson, Z. Kristallogr. 170, 225 (1985).

[11] R. H. Templer, J. M. Seddon, and N. A. Warrender, Biophys. Chem. 49, 1 (1994).

[12] J. F. Sadoc and J. Charvolin, Acta Crystallogr., Sect. A: Found. Crystallogr. 45, 10 (1989).

[13] Alto D. Benedicto and David F. O'Brien, Macromolecules 30, 3395 (1997).

[14] A. Fogden and S. T. Hyde, Eur. Phys. J. B 7, 91 (1999).

[15] G. E. Schröder, S. J. Ramsden, A. Fogden, and S. T. Hyde, Physica A 339, 137 (2004).
[16] H. Chung and M. Caffrey, Nature (London) 368, 224 (1994).

[17] U. S. Schwarz and G. Gompper, Phys. Rev. Lett. 85, 1472 (2000).

[18] Sérgio S. Funari, Burkhard Mädler, and Gert Rapp, Eur. Biophys. J. 24, 293 (1996).

[19] A. M. Squires, J. M. Seddon, and R. H. Templer, Langmuir 16, 3578 (2000).

[20] J. Erbes, R. Winter, and G. Rapp, J. Chem. Soc., Faraday Trans. 100, 1713 (1996).

[21] R. H. Templer, J. M. Seddon, N. A. Warrender, A. Syrykh, Z. Huang, R. Winter, and J. Erbes, J. Phys. Chem. B 102, 7251 (1998).

[22] A. M. Squires, R. H. Templer, J. M. Seddon, J. Woenckhaus, R. Winter, S. Finet, and N. Theyencheri, Langmuir 18, 7384 (2002).

[23] A. Schoppe, H.-J. Hinz, R. Gerdes, H. Redlich, and G. Rapp, Chem. Phys. Lipids 103, 95 (1999).

[24] H. G. von Schnering and R. Nesper, Z. Phys. B: Condens. Matter 83, 407 (1991).

[25] P. J. F. Gandy, S. Bardhan, A. L. Mackay, and J. Klinowski, Chem. Phys. Lett. 336, 187 (2001).

[26] G. E. Schroder, S. J. Ramsden, A. G. Christy, and S. T. Hyde, Eur. Phys. J. B 35, 551 (2003).

[27] http://www.esrf.fr/exp_facilities/ID2/handbook/handbook/ beamline.htm\#ID02 